\title{
New-Onset ANCA-Associated Vasculitis in a Patient with SARS-COV2
}

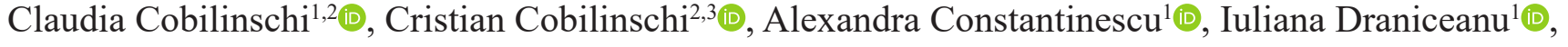 \\ Ruxandra Ionescu ${ }^{1,2}$ (D)
}

${ }^{1}$ Department of Rheumatology and Internal Medicine, Sf. Maria Clinical Hospital, Bucharest, Romania

${ }^{2}$ Carol Davila University of Medicine, Bucharest, Romania

${ }^{3}$ Department of Anesthesiology and Intensive Care, Bucharest Clinical Emergency Hospital, Bucharest, Romania

To the Editor,

A 67-year-old female with a medical background of hypertension and dyslipidemia was admitted with painful necrotic ulcerlike lesions on both legs, with perilesional edema and erythema (Figures 1 and 2).

Her medical history revealed the onset of the disease approximately one month before presentation, with polyarthralgia, lower-limb paresthesia, and palpable purpura, which gradually expanded. The appearance of the skin lesions was closely followed by sudden complete vision loss in the left eye, attributed to central retinal artery occlusion through both new-onset atrial fibrillation and high blood pressure values refractory to previous chronic medication. Ten days later, the patient experienced fever, dyspnea, and mild cough, and was immediately diagnosed with

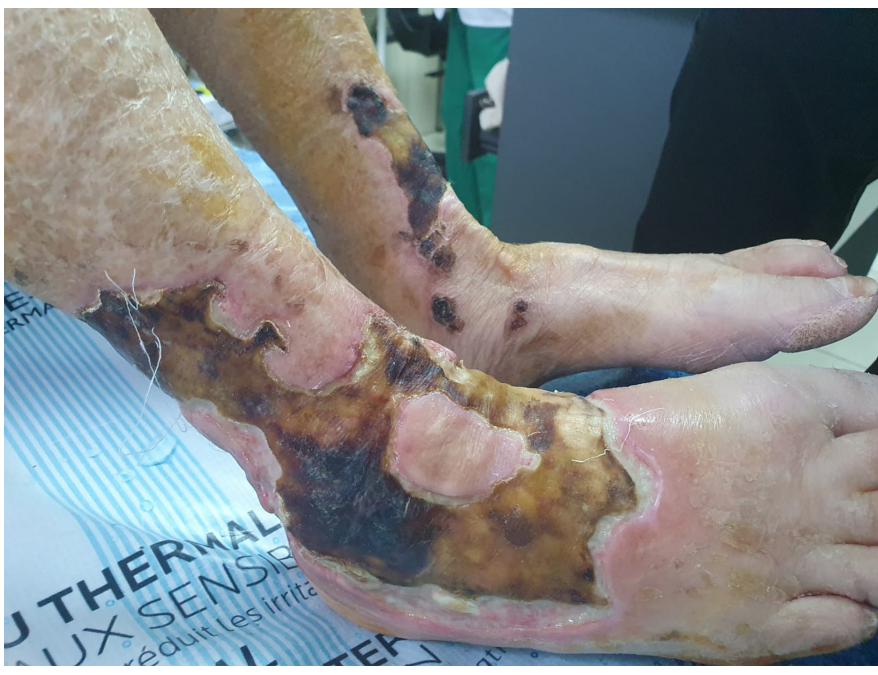

FIG. 1. Extensive ulcerative-necrotic lesions on leg and dorsal area of feet

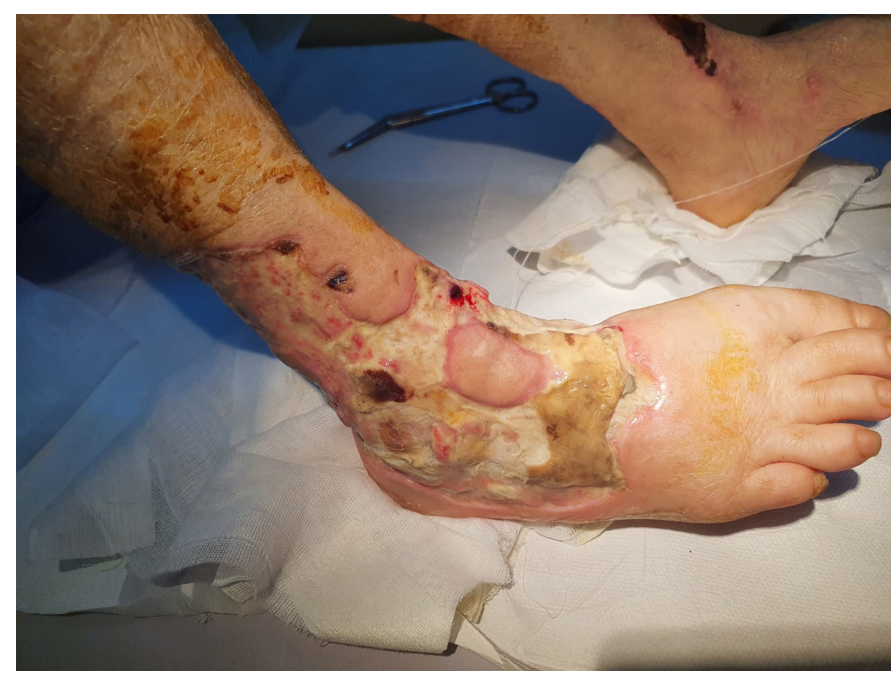

FIG. 2. Surgical debridement necrotic areas

SARS-COV2 infection through a real-time PCR test. A thoracic CT scan revealed left-sided ground glass opacity and pleural effusion, while head CT and Doppler ultrasound were normal, excluding giant-cell arteritis despite suggestive initial presentation.

Upon admission to the Rheumatology Department, laboratory tests revealed moderate normochromic normocytic anemia, markedly elevated inflammatory markers, and impaired renal function (serum creatinine $2 \mathrm{mg} / \mathrm{dl}$, stage 3 renal failure). Urinalysis showed significant proteinuria $(1 \mathrm{~g} / 24 \mathrm{~h})$ and hematuria, raising concerns of possible glomerulonephritis. Infection markers and cryoglobulins tested negative. Immunological tests revealed high-titer pANCA (5-fold reference laboratory value), antinuclear antibodies (ANA $1: 80$, homogenous pattern) and rheumatoid factor $(89 \mathrm{UI} / \mathrm{mL})$. Skin biopsy showed marked neutrophilic infiltrate of small vessels and area of necrosis suggesting vasculitis (Figure 4). Pyoderma

Corresponding author: Cristian Cobilinschi, Carol Davila University of Medicine, Bucharest, Romania; Department of Anesthesiology and Intensive Care, Bucharest Clinical Emergency Hospital, Bucharest, Romania

e-mail: klausini vs@yahoo.com

Received: May 26, 2021 Accepted: August 2, $2021 \cdot$ DOI: 10.5152/balkanmedj.2021.21129

Available at www.balkanmedicaljournal.org

ORCID iDs of the authors: C.C. 0000-0002-9117-2187; C.C. 0000-0002-1142-7427; A.C. 0000-0003-0043-4282; I.D. 0000-0001-8433-2069; R.I. 0000-0001-7651-0099

Cite this article as:

Cobilinschi C, Cobilinschi C, Constantinescu A, Draniceanu I, Ionescu R. New-onset anca-associated vasculitis in a patient with SARS-COV2. Balkan Med J. 2021;38(5):318-320.

Copyright@Author(s) - Available online at http://balkanmedicaljournal.org/ 


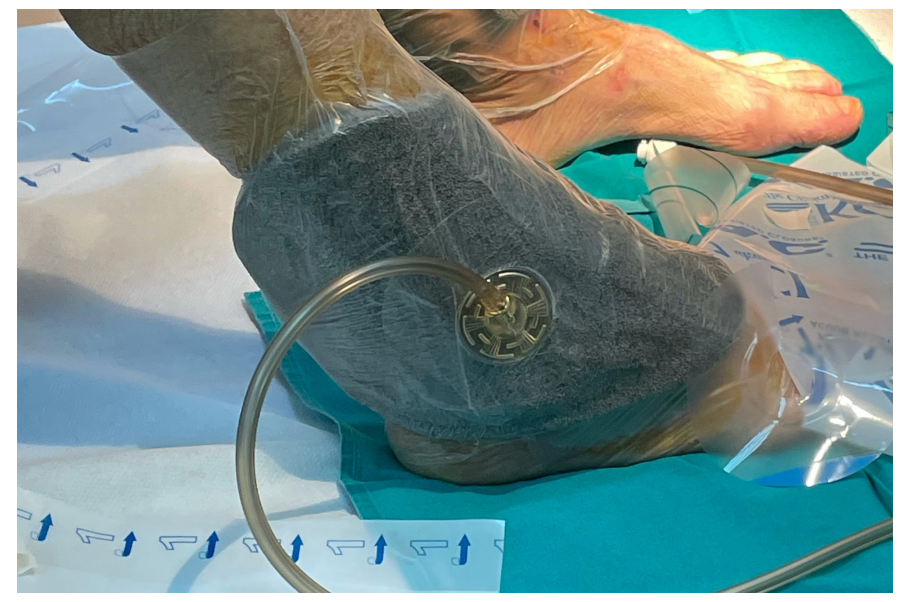

FIG. 3. Vacuum-assisted closure device on patient's lesion

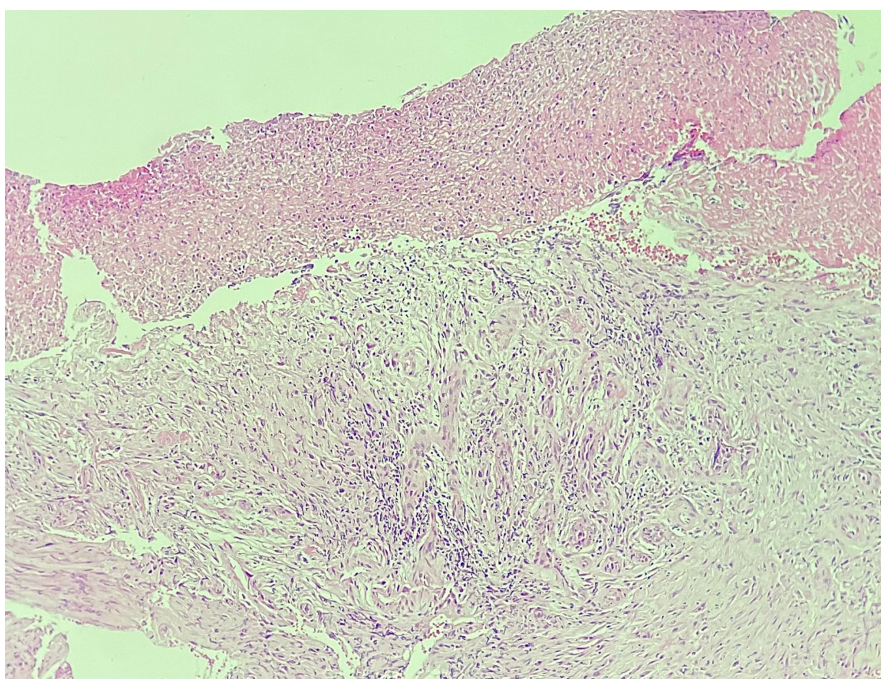

FIG. 4. Histopathological examination showing marked neutrophilic infiltrate

gangrenosum was ruled out due to histopathological details, lack of triggering factors, and insufficient response to glucocorticoids alone. The wound culture revealed an associated methicillin-resistant Staphylococcus aureus infection, with prompt initiation of appropriate antibiotic therapy.

The diagnosis of ANCA-associated vasculitis (AAV) with COVID-19 as trigger was established and treated with pulse corticosteroids, and monthly cyclophosphamide was initiated after confirming a negative COVID PCR result, with no new skin lesions, no added organ involvement, and reduction in antibody titer. Patient benefited from vacuum-assisted therapy (Figure 3 ) and later successfully grafted (Figures 5 and 6), with regain of mobility and increase in self-care management.

Since the beginning of 2020, the COVID-19 pandemic has been at the forefront of medical attention and research, with great strides being made in understanding this condition. By now, the typical disease presentation of fever, cough, and respiratory distress, along

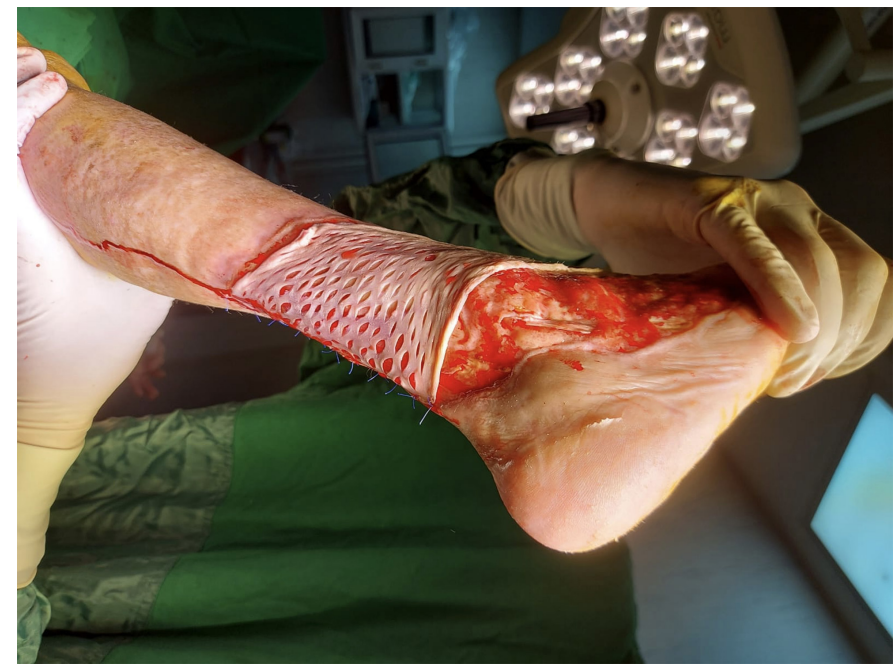

FIG. 5. Intraoperative image while grafting procedure

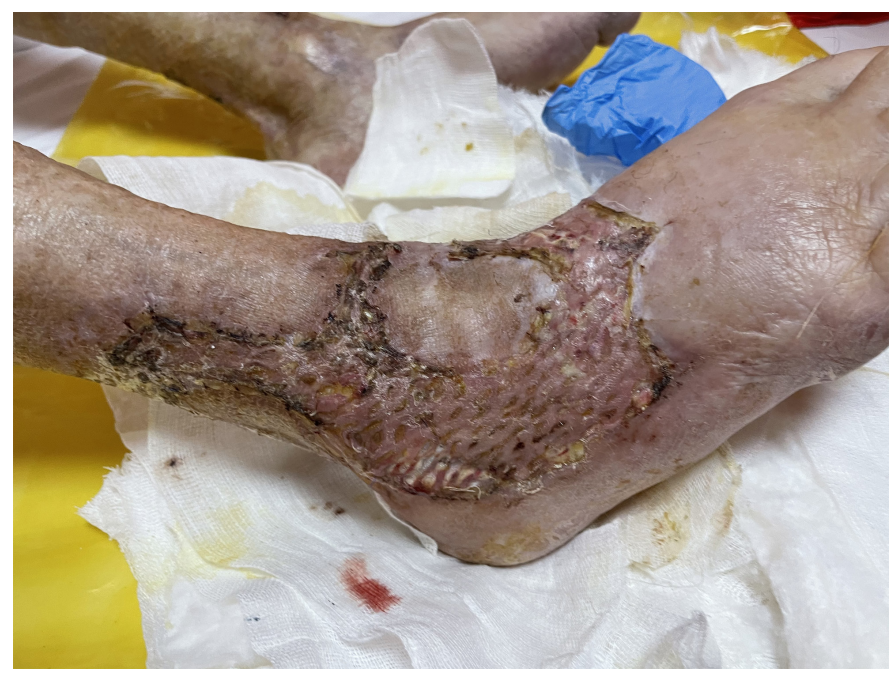

FIG. 6. Healing graft within recovery interval

with the predisposition toward thrombotic events, is familiar to all medical staff. However, atypical signs and symptoms are not uncommon, and the possible connection between this viral infection and vasculitis should not be ignored.

Vasculitis has been reported in an increasing number of COVID-19 patients, suggesting the need for further research. ${ }^{1,2}$ Though the above-presented case is not an isolated incident of vasculitis occurring in a COVID-19 patient, it is, to our knowledge, the one developing the most severe skin involvement. ${ }^{3,4}$

The COVID-induced cytokine storm and dysregulation of the immune system might have caused the onset of AAV but the link remains uncertain. The almost simultaneous onset of infection symptoms and purpura and the rapid worsening of skin lesions in parallel to the evolution of the respiratory disease strongly suggest a correlation between the two entities, with favorable response to immunosuppressive therapy. 
Patient Consent for Publication: Written informed consent was obtained from the patient.

Author Contributions: Concept - C.B., C.C.; Design - C.B., C.C.; Supervision - R.I.; Materials - C.C.; Data Collection and/or Processing - A.C., I.D.; Literature Review - A.C., I.D.; Writing - C.C.; Critical Review - R.I.

Acknowledgments: The authors thank Ruxandra Ciofu, MD Plastic, and reconstructive surgery, Constantin-Ioan Busuioc, MD Pathology.

Conflict of Interest: The authors has no conflict of interest to declare.

\section{REFERENCES}

1. Rehman S, Majeed T, Ansari MA, Al-Suhaimi EA. Syndrome resembling Kawasaki disease in COVID-19 asymptomatic children.JInfect Public Health. 2020;13(12):1830 1832. [CrossRef]

2. Becker RC. COVID19associated vasculitis and vasculopathy. J Thromb Thrombolysis. 2020;50(3):499-511. [CrossRef]

3. Kösters K, Schwarzer S, Labuhn A, et al. Cutaneous vasculitis in a patient with COVID-19. Open Forum Infect Dis. 2020;7(10):ofaa474. [CrossRef]

4. Uppal NN, Kello N, Shah HH, et al. De novo ANCA-associated vasculitis With glomerulonephritis in COVID-19. Kidney Int Rep. 2020;5(11):2079-2083. [CrossRef] 